\title{
SEQUENCING OF ORE COLUMNS FOR PLANNING OF LARGE UNDERGROUND MINES
}

\author{
Hylke J. GLASS ${ }^{1}$ * \\ Gert van HOUT $^{2}$ \\ ${ }^{1}$ University of Exeter, Camborne School of Mines, Penryn TR10 9FE, UK \\ 2 Rio Tinto, 6 St James's Square, London SW1Y 4AD, UK
}

\begin{abstract}
Block caving is an underground mining technique which extracts ore from the base, rather than from the top, of typically massive deposits. Mining infrastructure is developed below the deposit before extraction commences. A network of tunnels provides access to a collection of drawpoints from which ore is mucked and hauled. With large deposits, not all drawpoints are developed simultaneously and the opening of drawpoints is sequenced to facilitate orderly extraction of ore columns above drawpoints. Sequencing fixes the initiation point for the entire block cave, or a part of it, as well as identifying the direction of cave advancement. The sequence of opening drawpoints influences the mine economics and is an integral aspect of planning a block cave mine. This paper discusses the optimisation of sequencing based on the net present value associated with extraction over the life-of-mine. It is shown that the maximum attainable net present value is obtained by a sequence in which ore columns are ranked in descending order of value. If significant variation of grade is present inside columns, an iterative procedure is given which corrects the sequence which yields the maximum net present value. The sequence with maximum net present value may not be practical or attractive from a caving perspective. Systematic design of sequences which permit orderly development of a block cave is discussed. To provide context, the net present value obtained from these feasible sequences is compared with the maximum attainable net present value. It is shown that the best feasible sequences in terms of net present value are preferentially initiated in zones with columns of high-grade ore.
\end{abstract}

Keywords: block caving, sequencing, drawpoints, optimisation, net present value

* Corresponding author: H.J.Glass@exeter.ac.uk (H.J. Glass)

doi: $10.37190 / \mathrm{msc} 192613$ 


\section{INTRODUCTION}

As global demand for metals is continually rising, the number of large open pit mines is more or less stable in recent years. This could be due to scarcity of large, nearsurface deposits, which may herald a shift towards development of deep underground orebodies. Traditionally, underground mining has focussed on selective extraction of high-grade ore in relatively modest tonnages. Going forward, mining operations will seek to extract ore from massive underground orebodies in tonnages comparable to those realised with open-pit mining. In order to achieve this objective, cost-competitive mining methods and associated technology and equipment are emerging.

A notable example of a large-scale underground mining method is block caving. With this technique, ore is drawn down to an underground extraction level before being transported to surface. While the operational unit cost of extracting ore with block caving is attractive by underground mining standards, the method requires significant upfront capital expenditure prior to the start of mining. This is primarily related to the development of underground infrastructure which, for example, includes the sinking of shafts to depth and creating an intricate network of tunnels below the deposit. The extent of the tunnel network matches the footprint of the deposit and provides access to numerous drawpoints. At a drawpoint, broken ore is collected from a slot below a drawbell. The flow of ore into an individual drawbell is initiated by undercutting the orebody, i.e. pre-blasting the rock above a drawpoint.

In practice, the collection of ore from drawpoints is carefully planned to optimise the extraction of ore within strict geotechnical-informed guidelines. With large orebodies, drawpoints are sequentially opened in a sequence determined during mine planning. Once mining commences, the flexibility to adjust the sequence of opening drawpoints is limited. This paper addresses the question how to define an optimal drawpoint opening sequence during mine planning and discusses a novel approach to provide a practical solution.

\section{DRAWPOINTS AND ORE COLUMNS}

When exploration of the underground progresses to an order-of-magnitude study, the deposit is typically discretised into a collection of stacked blocks and relevant properties are assigned to each block. Geochemical and mineralogical data is typically obtained from the analysis of sections of drillcores extending into the deposit. While the spacing of measured data generally exceeds the block size, block properties are routinely estimated with geostatistical techniques. Once block properties or attributes are defined, columns of block grades are migrated to a pattern of drawpoints at a fixed elevation. The layout of drawpoints on the extraction level may differ significantly 
from the block model. Identifying an appropriate arrangement of drawpoints requires understanding of ore caveability and primary fragmentation (Laubscher, 1994), as well as envisaged production targets (e.g., Rafiee et al. 2018).

When the ore associated with each drawpoint is identified, the economic value associated with a drawpoint can be determined. The value of a column of ore consists of revenue from commodities contained in the column minus the cost of extracting the column. In the first instance, all columns with sufficient economic value are considered in developing a mine plan. The economic value of an ore column also depends on the timing of its extraction. A popular measure to express time-based is the Net Present Value (NPV). Considering that grade may vary in the blocks which make up the column, the NPV will calculated as follows from the value of blocks:

$$
\mathrm{NPV}=\sum_{t=1}^{T} \frac{\sum_{i=1}^{S} \theta_{i}}{(1+r)^{t}}
$$

where:

$T$ is the life of mine expressed in years,

$S$ is the number of blocks mined (in part) in year $t$,

$\theta_{i}$ is the net value of block $i$, and

$r$ is the discount rate.

To calculate the NPV, insight into the timing of access to, and extracting, a block is required. The timing is informed by the location of blocks in a column and the time when extraction from the column commences, i.e. when the drawpoint is opened. In practice, it may be prove challenging to mine according to the envisaged NPV. However, NPV is a useful measure to optimise the sequence of opening drawpoints in relation to the orebody properties, as well as mining and geotechnical considerations. As a first step, the definition of a footprint containing columns to be sequenced should be addressed.

A basic approach to establish column height compares the cut-off grade relating to a property of interest with the estimated block grade, starting from the highest elevation and working downwards. The upper limit of a column is defined by the elevation where the cut-off grade is exceeded in a block for the first time (true height given by green line in Fig. 1, right). Alternatively, the upper limit of a column may be constrained by the maximum height which is deemed appropriate by mining engineers (maximum height given by blue line in Fig. 1, left). The lower limit of a column is defined by the elevation of the extraction level. If the cut-off grade is not exceeded in any block above the extraction level, the column is discarded from the mining footprint. If the upper limit is defined by a relatively isolated block, i.e. surrounded by blocks consisting of waste, this approach could lead to mining of too much waste. 


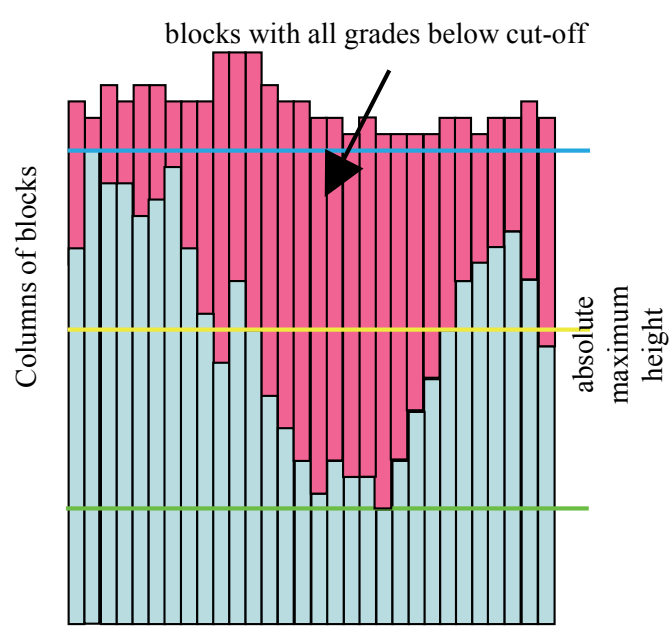

Eastings or Northings

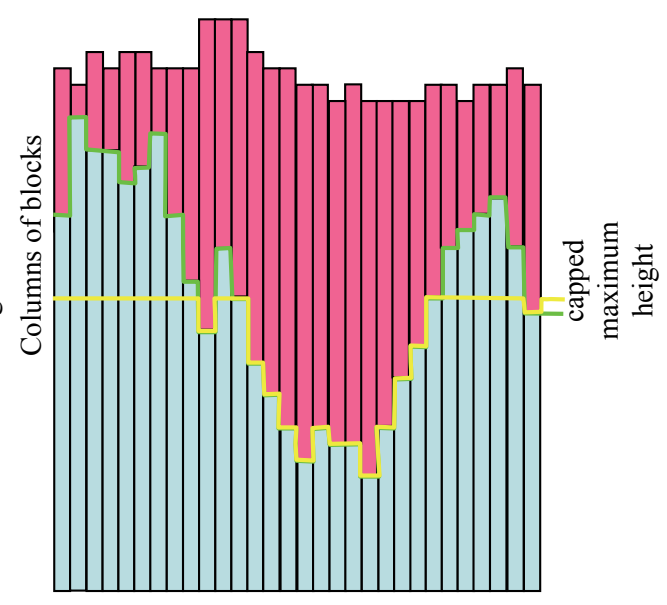

Eastings or Northings

Fig. 1. Selected options for definition of column height above the extraction level

A rigorous approach evaluates the inclusion of any column by calculating the column NPV, starting at the base of the column and working upwards. The upper elevation of the column is determined by the column height which is associated with the maximum NPV. If the maximum NPV fails to reach a minimum value, the column is not considered part of the mining footprint. When this approach leads to large differences in the height between adjacent columns in the interior of the footprint, a minimum column height or a less steep transition may need to be imposed.

Originally, block caving was envisaged for columns with a height of about $150 \mathrm{~m}$. For deposits with a larger vertical dimension, the concept of a single extraction level below the deposit requires much confidence in the caving mechanics of tall ore columns, reduces the flexibility of caving to align economically with the block grade distribution, and increases the necessary upfront capital expenditure. In the case of very tall ore columns, the staged extraction through a series of levels, or lifts, may be attractive. Note that the height of columns below the upper lift is necessarily fixed by the vertical distance between successive deeper lifts. The size, location, the elevation of each lift may be adjusted to add value and reduce risk. Determination of the best sequence in which drawpoints should be opened in each lift is described next.

\section{MAXIMUM NET PRESENT VALUE}

For a given lift, a brute force approach to identifying a sequence which is optimal, i.e. generates a maximum NPV, is to calculate and compare the NPV of every feasible 
sequence. This approach soon becomes computationally onerous because a footprint may contain hundreds of drawpoints which can be arranged in the factorial of $\mathrm{N} \mathrm{se}$ quences, where $\mathrm{N}$ is the number of drawpoints. It is, however, instructive to consider the outcome of such an exercise because knowledge of the maximum NPV puts perspective on the mining proposition. It is possible to establish the sequence which produces the absolute maximum NPV without calculating all possible sequences: provided that the distribution of value inside the columns is uniform, the maximum NPV is always associated with a sequence where drawpoints are opened in descending order of value. Assuming that a single ore column is assigned to each drawpoint, the maximum NPV follows from:

1) determine the value of individual columns, with every column being opened at the same time $(t=0)$,

2) rank the columns according to their value,

3) equate the best sequence to the ranking of columns in order of descending value,

4) determine the NPV of the sequence of ranked columns.

When zones of distinct grade exist within columns, the effect of grouping drawpoints during time periods defined by the NPV calculation may affect the sequence which provides the maximum NPV. Figure 2 shows that the sequence with columns ranked according to average grade is associate with a lower NPV than a sequence where the effect of time is taken into account.

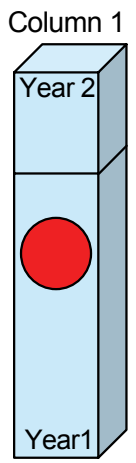

First column to be opened

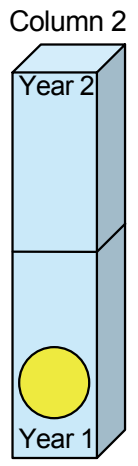

Second column to be opened

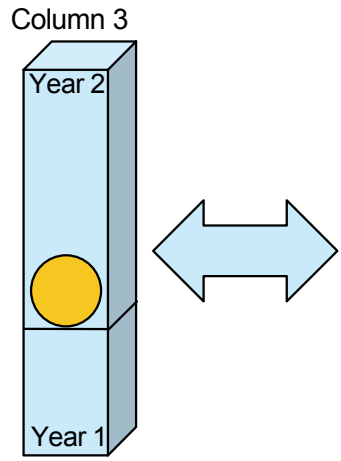

Third column to be opened

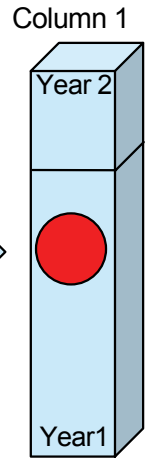

First column to be opened

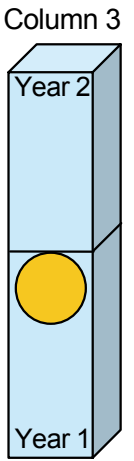

Second column to be opened

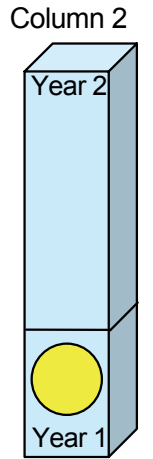

Third column to be opened

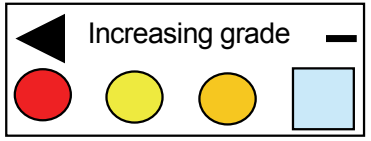

\section{Sequence 1-2-3 \\ Sequence 1-3-2 \\ NPV < \\ NPV}

Fig. 2. Comparison of NPV of columns sequenced according to descending average grade (left) and maximum NPV (right) 
To mitigate for the effect of timing, a correction to the - up to now - best sequence may be required. This entails an iterative process which works through the columns in the sequence, starting from the first column, i.e. with the highest value. Figure 3 shows a flowchart of the sequence correction process.

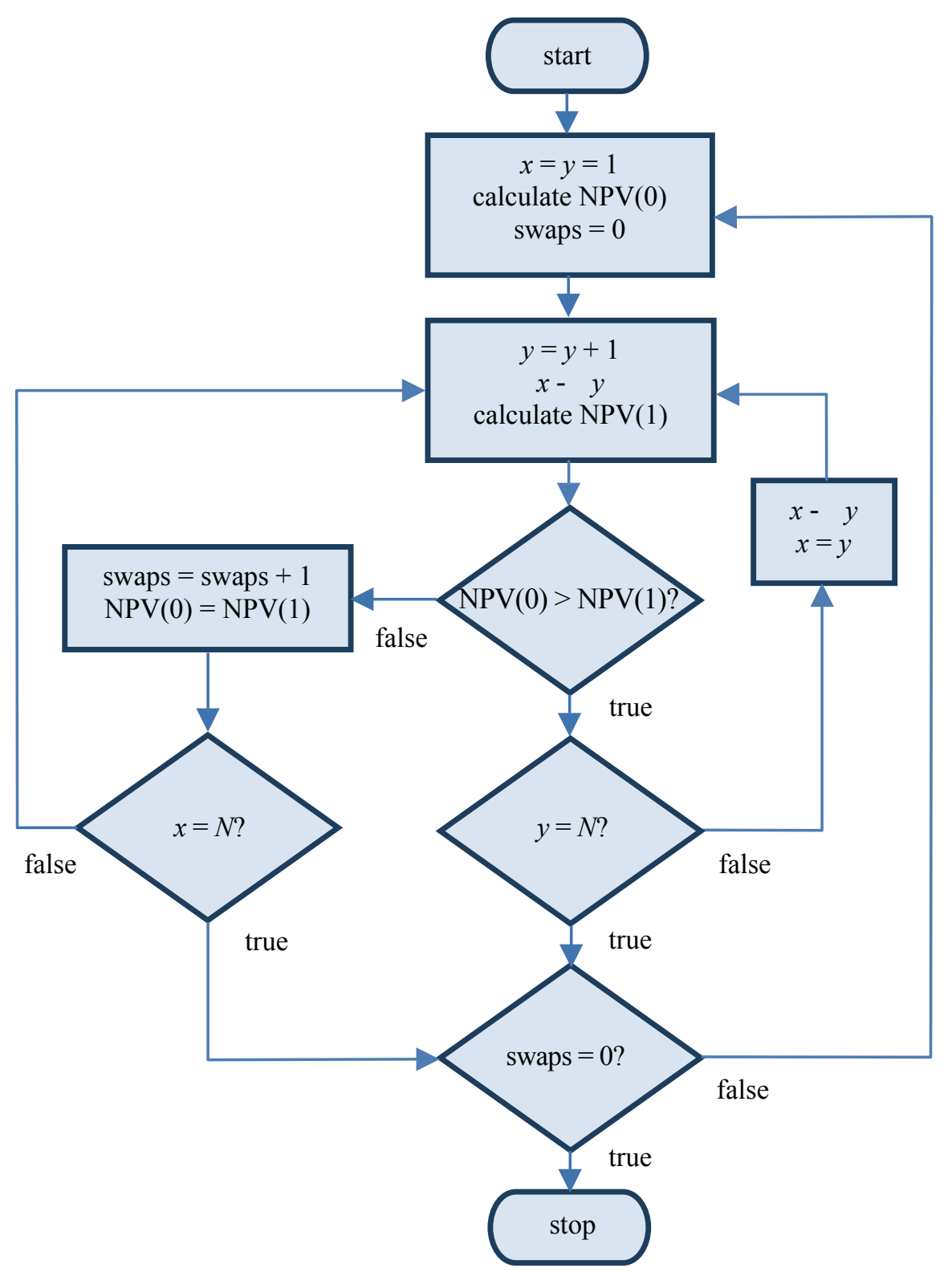

Fig. 3. Method for calculating the true maximum net present value, given by NPV(0) 
The highest value column is provisionally exchanged with the second highest value column and the effect on NPV is determined. If the NPV increases, the column exchange is made permanent and the process considers the exchange of the initially highest value column with the column with the third highest value. This process continues until no improvement in NPV is observed or the column with the lowest value in the footprint is reached. When no improvement in NPV is observed, the last column becomes the new starting column for an exchange process with lower value columns. When the column with the lowest value in the footprint is reached and column swaps were made during that iteration, the exchange process is re-started with the top-ranked column in the sequence. The process terminates when no swaps were made during the last iteration. As a general rule, the number of swaps observed during an iteration will decrease as the number of iterations increases while the improvement in the maximum NPV will level off. Figure 4 illustrates these complementary trends for an example.
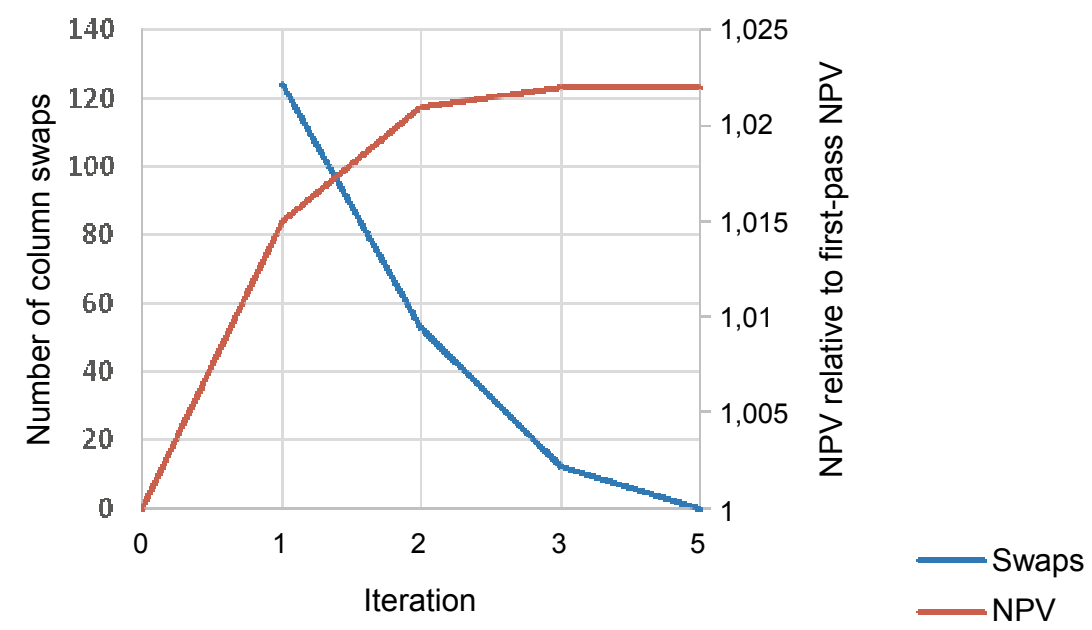

Fig. 4. Correction of the sequence based on ranking columns according to descending value

Once the sequence which yields the maximum NPV has been defined, it is instructive to determine whether this sequence allows orderly development of a block caving operation from a practical and geotechnical perspective. This requires attention to the development of caving front in a way which allows for steady ore flow and suitable secondary ore fragmentation while horizontal mixing between, and vertical mixing within, columns is minimised. A further consideration is the compatibility of the best sequence with efficient scheduling of ore haulage. In view of the important but limited significance of NPV as a criterion for identifying the best sequence, a modified strategy is proposed. The first step is to develop a systematic review of sequences which are deemed to be feasible and practical for a given min- 
ing footprint at a prescribed elevation. The second step is to determine the NPV of these feasible sequences and rank the sequences according to NPV. The third step is to analyse and compare the top-ranked sequences, which are likely to have similar NPV, in terms of feasibility.

\section{FEASIBLE SEQUENCE DESIGN}

While ore comminution is an energy-intensive process, block caving owes part of its promising economics to induced fragmentation of ore during gravity flow of ore into drawbells. After a drawpoint is opened and ore flow has commenced, the collected ore originates mainly from a virtual silo directly above the drawbell. To achieve good draw control, strict compliance to rigid draw rules is enforced. In practice, the flow of ore into a drawbell produces point load forces between rock blocks and shear stresses with ore surrounding the silo. While these forces and stresses can create additional fragmentation in the surrounding ore, the greatest benefit is observed if downward flow of a column of ore is initiated from movement of ore in a neighbouring column. This suggests that block caving is not optimal when extracting isolated columns of ore.

Feasible sequences are defined as those where a succession of neighbouring drawpoints are opened. Taking this to its extreme, the opening of drawpoints would commence below the centre of the deposit and radiate in all directions until reaching the boundaries of the footprint. In practice, the benefit of enhanced ore fragmentation above adjacent drawbells can only be realised for a limited period of time. This implies that, in a feasible sequence, the time delay between opening adjacent columns is at most equal to a specified value. With a view to identifying the optimum feasible sequence, a systematic approach is adopted, from designing basic sequences to more sophisticated variants.

Starting point for sequencing is the availability of a block model which encompasses the entire orebody. The block model is superimposed on a grid of drawpoints in a two-dimensional footprint. While the shape of the footprint will be subject to optimisation, it is likely to feature corners and edges. Figure 5 shows how feasible sequences can be designed in a systematic way.

The most basic sequences are those which are initiated from a footprint corner. Further columns are successively opened along a series of parallel transects. These transects are always perpendicular to the direction of the mining front and mining advances across the footprint at a fixed orientation. A V-shaped sequence may also be initiated from a footprint corner to gain faster access to relatively rich ore columns located about the centre of the footprint. When ore columns in the vicinity of footprint corners contain relatively lean ore, initiation from the footprint edge may be advantageous in terms of NPV. Sequences initiated from the footprint boundary are typically 
V-shaped and traverse the footprint by interleaving the opening of columns on either front.

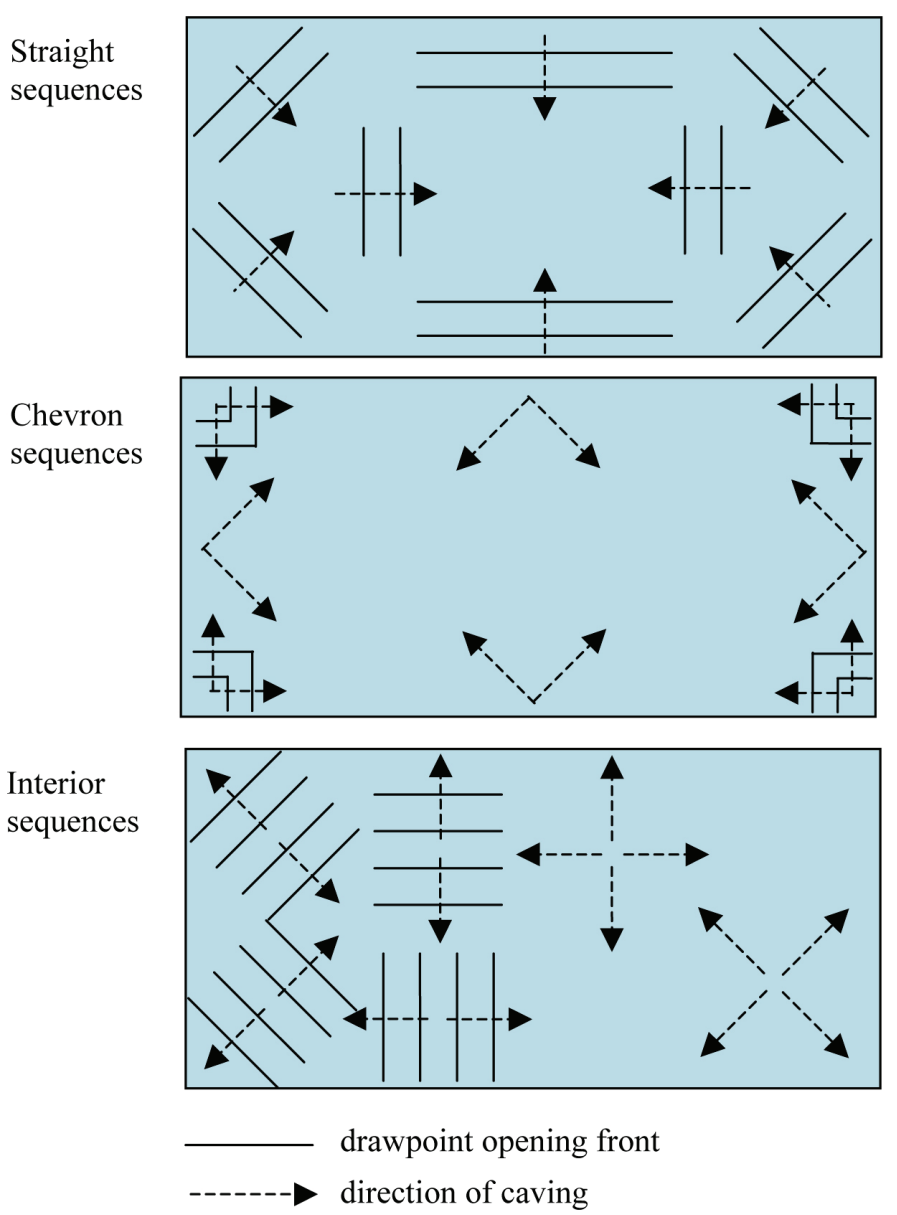

Fig. 5. Systematic conceptual design of feasible sequences

Variants of sequences starting from the footprint perimeter can preferentially open columns along the centreline or along the flanks on either side of the centreline. In all basic sequences, the opening of columns can be described as clockwise, anticlockwise, or alternating between these about the direction in which mining advances across the footprint. Based on the distribution of columns of relatively rich ore in the footprint, there may be a case to commence mining from columns inside the interior of the footprint. Basic sequences initiated inside the footprint may consist of two fronts moving in opposite directions at a fixed orientation across the footprint. Note that during simultaneous advance of both fronts, the opening of columns in either front is inter- 
leaved. In cases where columns with rich ore are concentrated in the core of the footprint, a radial opening pattern is attractive. Such sequences feature a diamond shape with two $\mathrm{V}$-shaped fronts advancing in opposite directions from the column where mining was initiated. The application of these sequences is explored with an example in the next section.

\section{SEQUENCE NPV AND GRADE DISTRIBUTION}

For a given footprint, there are many feasible sequences which enable the orderly development of one or more caving fronts. Once feasible sequences are classified according to relative NPV, further analysis of the top-ranked sequences is required to determine which sequence is the most appropriate. An important characteristic of the selected sequence is the location of the drawpoints where block caving is initiated. While the sequence develops about the cave initiation point, the cave initiation point will be linked to patterns in the distribution of grade within the deposit. To illustrate this, a hypothetical orebody is studied which features a relatively high-grade core and a radial decrease of grade around the core. Figure 6 shows a plan view of the grade distribution.

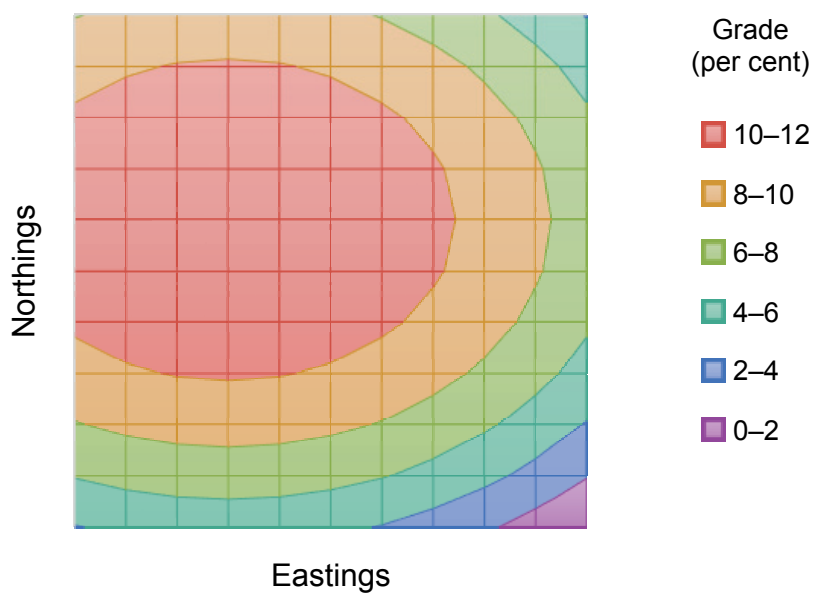

Fig. 6. Grade distribution within a section of a hypothetical deposit

A mining footprint is defined for this section of the orebody, which is assumed to extend vertically with the same grade. Ore columns are defined with a fixed height and a constant, average grade. Figure 7 shows the average grade assigned to ore columns while the location of drawpoints is indicated by circles. It should be noted that the average grade is proportional to the column value. 


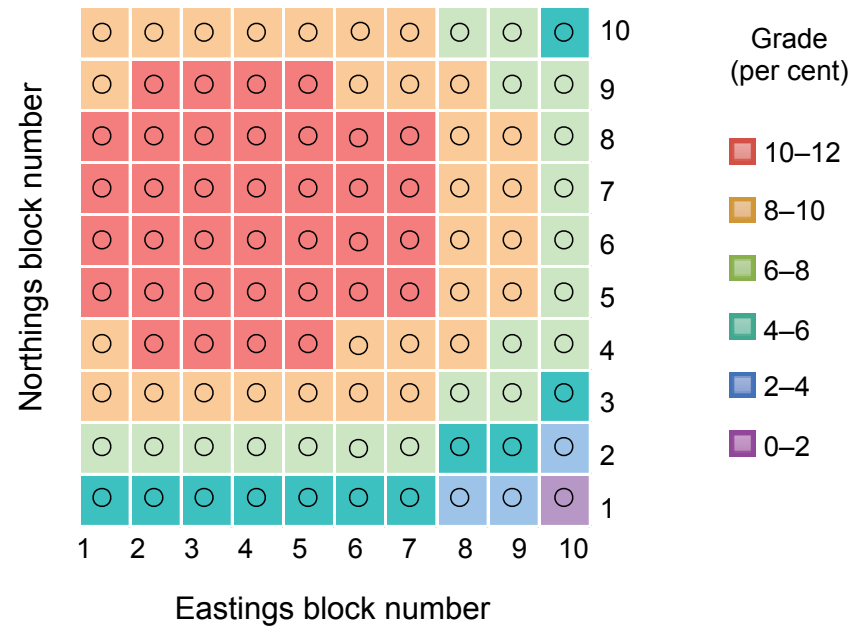

Fig. 7. Column value and drawpoint layout for block caving example

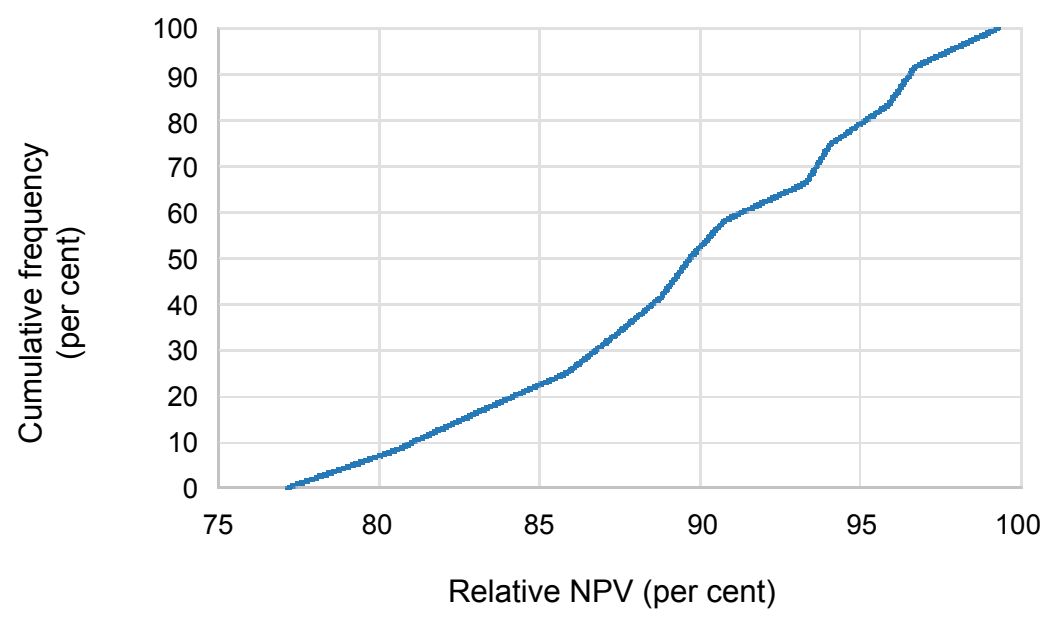

Fig. 8. Variation of relative NPV for feasible sequences

The 100 drawpoints in the footprint will be opened at a rate of 10 per year while the annual discount rate is fixed at 10 per cent. A total of 1592 feasible sequences are characterised in terms of the relative NPV, which is the percentage of the maximum attainable NPV. Figure 8 plots the cumulative frequency of the relative NPV of these sequences, highlighting that sequencing has a significant effect on NPV in this example.

A closer examination of sequences ranked according to NPV shows that the optimum sequence reaches a relative NPV of 99.27 per cent by opening drawpoints in a sequence which expands according to a diamond-shaped pattern during 19 predefined time periods (Fig. 9). 


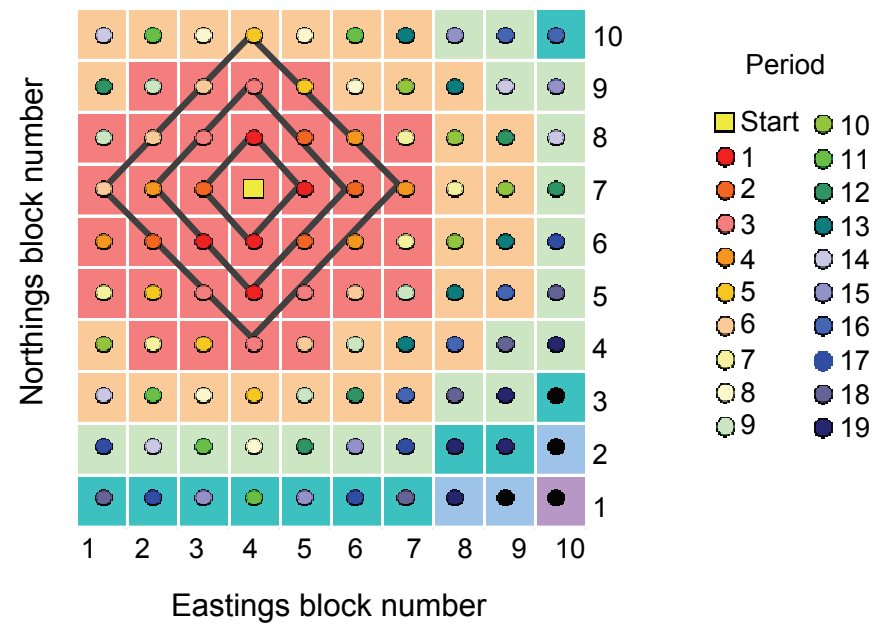

Fig. 9. Optimal sequence for opening drawpoints (relative NPV $=99.27$ per cent)

The drawpoint where caving is initiated is more or less in the centre of the high grade zone, with a marginal preference for the upper half of the footprint. The latter is explained on the basis of a delay in opening drawpoints in the lower half of the footprint. This suggests that sequencing according to NPV is highly sensitive to the distribution of value in footprint columns. If cave initiation would start in the lower half of the footprint, where column values are generally lower, a significant reduction in NPV is observed. For example, starting the opening of drawpoints in an expanding chevron pattern, from the lower edge of the footprint, reduces the relative NPV to 82.81 per cent (Fig. 10).

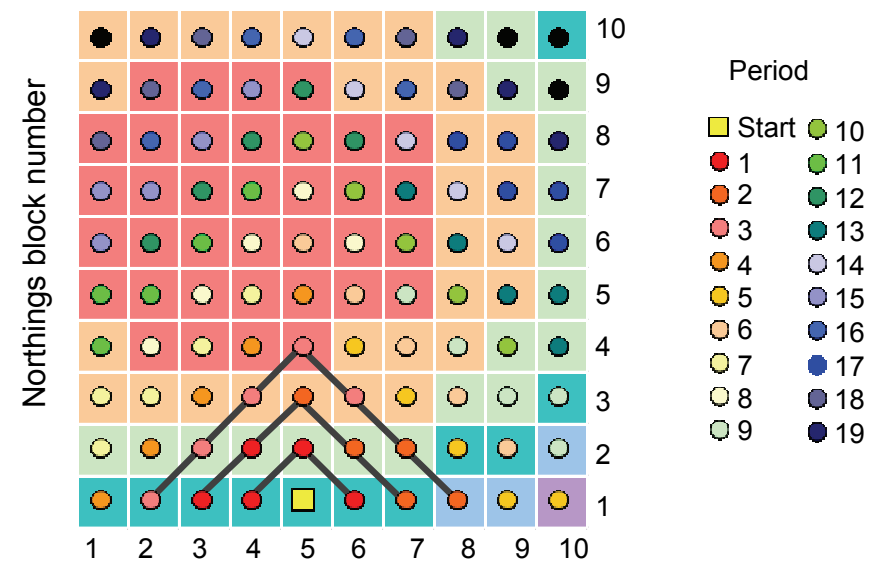

Eastings block number

Fig. 10. Chevron sequence for opening drawpoints (relative NPV $=82.81$ per cent) 
Although the sequence shown in Fig. 10 appears to be an unattractive option, practical and geotechnical considerations often force the mine design to implement a chevron starting at the footprint boundary. The orientation of geological features and the in situ stress distribution has a significant impact on the geotechnical stability and safety conditions of the working areas. As a result, the opening sequence of choice needs to be at an oblique angle to the strike of geological features and as parallel as possible to the major horizontal in situ stress component.

While the shape of the caving front has some influence on the relative NPV, the link with the underlying value distribution in the footprint is possibly the most significant. For example, if the expanding chevron pattern is initiated close to the high-grade core on the left edge of the footprint, the relative NPV rises sharply to 98.43 per cent (Fig. 11).

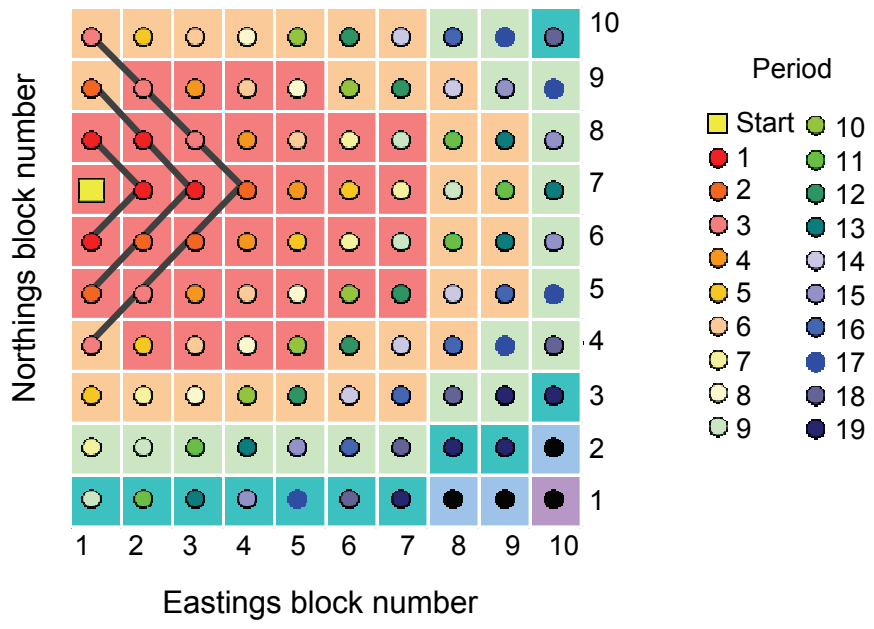

Fig. 11. Chevron sequence for opening drawpoints (relative NPV $=98.43$ per cent)

When different sequences commence from the same drawpoint, some influence of the direction in which the caving front develops on the relative NPV can be discerned. As shown in Fig. 12, sequences for a caving front moving downwards and a caving front moving across the footprint from the same initiation point yield a relative NPV of 93.00 per cent and 93.52 per cent, respectively.

Even when the caving front develops from a fixed starting point in the same direction, the exact specification of the opening sequence can still have subtle impact on the relative NPV. For deposits which contain a number of high-grade zones, the optimum sequence will necessarily represent a compromise in terms of distance to access to high-grade ore zones. In such a case, partitioning the deposit and sequencing individual sections may prove beneficial. This also applies when the deposits has an irregular geometry or complex geology. 

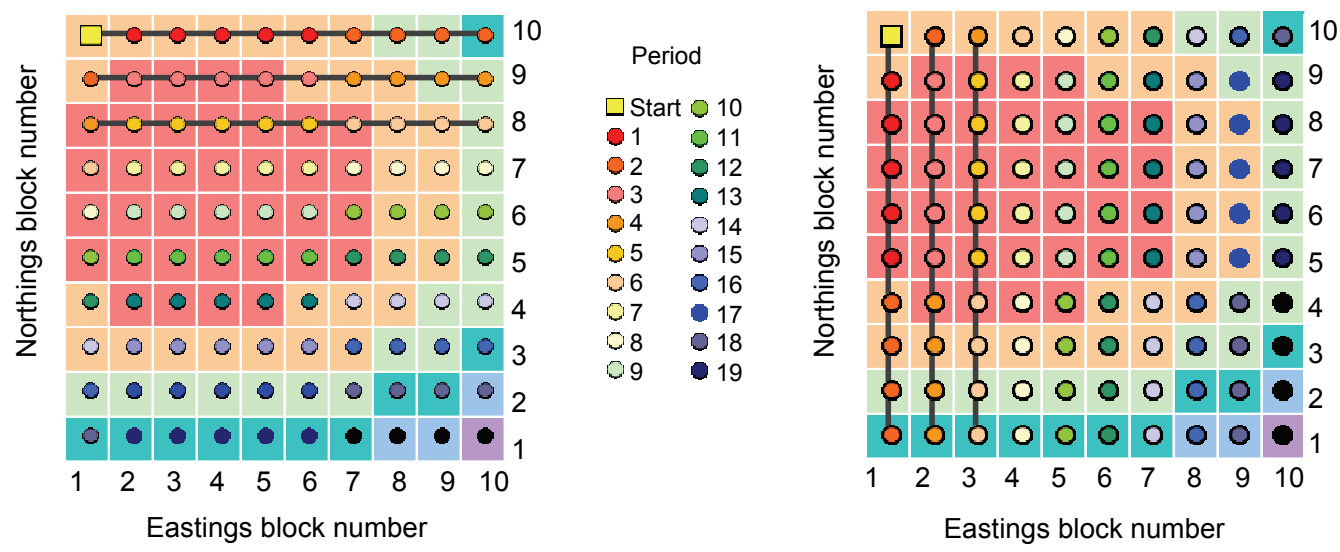

Fig. 12. Straight sequences for opening drawpoints. Relative NPV $=93.00$ per cent (left) and 93.52 per cent (right)

\section{CONCLUDING REMARKS}

By developing a set of feasible sequences, the example in the previous section shows that a sequence optimised for net present value of the mine tends to favour early extraction from zones with relatively high-value ore columns. Although relative NPVs are indicative only, observed differences in the example suggest that the location of the cave initiation point and the sequence developed about this point exert profound and material influence on life-of-mine economics, influencing development of both mining infrastructure, the timing of first ore, and operations. It is important to determine the optimal sequence of opening drawpoints in consideration of structural geology and the geotechnical environment while setting variables such as elevation of extraction level, lift height, footprint perimeter, rate of drawpoint opening, draw rate profile from individual drawpoints over time, direction of caving advancement, extent of vertical and horizontal mixing, and discount rate. Furthermore, catering for the effect of uncertainty in estimated block grades may have an effect on the optimal sequence.

\section{REFERENCES}

CACCETTA L., HILL S.P., 2003, An application of branch and cut method to open pit scheduling, Journal of Global Optimization, 27, 349-365.

DIMITRAKOPOULOS R., FARRELLY C., GODOY M., 2002, Moving forward from traditional optimization: grade uncertainty and risk effects in open pit design, Transactions of the Institution of Mining and Metalurgy section A, 111, A82-A88.

LAUBSCHER D.H., 1994, Cave mining - the state of the art, Journal of the South African Institute of Mining and Metallurgy, 94, No. 10, 279-293. 
LERCHS H., GROSSMAN F., 1965, Optimum Design of Open-Pit Mines, Transactions of the CIM, 58, 47-54.

KHALOKAKAIE R., DOWD P.A., FOWELL R.J., 2000, The Lerchs-Grossmann algorithm with variable slope angles, Transations of the Institution of Mining and Metallurgy Section A, 109, A77-A85.

RAFIEE R., ATAEI M., KHALOKAKAIE R., JALALI S.E., SERESHKI F., NOROOZI M., 2018, Numerical modelling of influence parameters in cavability of rock mass in block caving mines, International Journal of Rock Mechanics and Mining Sciences, 105, 22-27.

TROTTER D.A., GODDARD G.J., 1981, Design techniques for sublevel caving layouts, CIM Bulletin, $74,92-100$. 